\title{
Prevalence and Predictors of Exclusive Breastfeeding in Late Preterm Infants at 12 Weeks
}

\author{
Soo Yeon Lee ${ }^{1}$, Gun Ja Jang ${ }^{2}$ \\ ${ }^{1}$ Department of Nursing, Geoje College, Geoje; ${ }^{2}$ Department of Nursing, Daegu University, Daegu, Korea
}

Purpose: The purpose of this study was to identify breastfeeding practice with late preterm infants (LPIs), and to determine predictors of exclusive breastfeeding at the 12th week after discharge. Methods: The participants were 106 mothers of LPIs hospitalized in neonatal intensive care units at two university hospitals. Data were collected between February and October, 2013. Questionnaires included characteristics of LPIs, their mothers, and feeding-related characteristics. Feeding methods were exclusive breastfeeding, mixed feeding, and formula feeding. Results: Exclusive breastfeeding steadily increased from $5.7 \%$ at the 1 st week to $19.8 \%$ at the 12 th week, as did formula feeding from $27.3 \%$ to $67.9 \%$. Contrarily, mixed feeding decreased from $67.0 \%$ at the 1 st week to $12.3 \%$ at the 12 th week. The ratio of formula feeding was higher than that of exclusive breastfeeding over time. Predictors for exclusive breastfeeding were the following: type of delivery $(\mathrm{OR}=2.96,95 \% \mathrm{CI}=1.07-8.14)$, feeding intolerance $(\mathrm{OR}=3.03,95 \% \mathrm{CI}=1.26-7.25)$ and feeding method during hospitalization $(\mathrm{OR}=7.84,95 \% \mathrm{CI}=3.15-19.53)$. Conclusion: In order to increase breastfeeding opportunities for LPIs, educational programs for gestational age-appropriate breastfeeding should be developed. The focus of breastfeeding education needs to be on mothers who delivered their LPIs through Cesarean-section and LPIs who had feeding intolerance or were fed only formula during hospitalization.

Key words: Exclusive breast-feeding, Formula, Preterm infant

\section{Introduction}

Gestational age at birth is one of the major indicators used to assess newborn mortality and morbidity, and the World Health Organization classifies the maturity of newborns at birth into premature, full-term, and postmature[1]. Among the premature, late preterm birth is defined as birth at the gestational ages between 34 weeks and 36 weeks[2].

Statistics Korea classifies late preterm births in Korea as infants at the gestational ages between 32 and 35 weeks, or at 36 weeks, which makes it difficult to identify the exact number of newborns at the gestataional ages between 34 and 36 weeks. Premature births in Korea were estimated at 30,376 and among those premature newborns, the births at the

Corresponding author Gun Ja Jang Department of Nursing, Daegu University, 33 Seongdang-ro 50-gil, Nam-gu, Daegu 42400, Korea

TEL +82-53-650-8391 FAX+82-53-650-8399 E-MAIL kjjang14ddaegu.ac.kr

*This work was supported by the Korea Research Foundation (KRF) grant funded by the Korea government (MEST) (2009-0070334).

Received 7 March 2016 Received in revised form 11 April 2016

Accepted 15 April 2016

() This is an Open Access article distributed under the terms of the Creative Commons Attribution NonCommercial License (http://creativecommons.org/licenses/by-nc/3.0/) which permits unrestricted noncommercial use, distribution, and reproduction in any medium, provided the original work is properly cited. gestational ages between 32 and 36 weeks were estimated at 26,721, which is $88 \%$ of all the delivered premature babies in 2012[3]. The lack of concern and attention of health care providers may be due to the physical maturity and health condition of the late preterm infants being so similar to those of full-term infants, whereby the majority of late preterm infants (LPIs) are discharged from hospitals after receiving general medical and nursing care management tailored for full-term infants. However, the onset of breastfeeding for LPIs is delayed longer than that for fullterm infants[4], and the rates of breastfeeding at home at the 4th week after discharge appeared to be lower[5]. In addition, LPIs are more likely to be re-hospitalized due to health problems such as jaundice or other infectious diseases caused by mothers' inexperience or a lack of feeding techniques[6-9], all of which require health care providers to furnish the LPIs with more effective intervention for breastfeeding. Despite the fact that LPIs are the majority of premature newborns, there has been little research by healthcare providers in Korea[6,7,10,11] addressing breastfeeing for LPIs

There are several reasons why health care providers should focus on the promotion of breastfeeding for late preterm infants. First, the birth rate in Korea has steadily decreased over the past three or four decades, 
while the proportion of premature and late preterm newborns has consistently increased. This requires health care providers to have more concern for premature newborns. Second, the World Health Organization recommends that infants should be exclusively breastfed for the first six months[12], especially LPIs who did not receive immunological substances from the mother during the late phase of pregnancy due to premature labor. Breastmilk plays an essential role in preventing the late preterms which from getting infectious diseases[13]. Third, breastfeeding for LPIs depends on the infant's health condition, especially respiratory problems which induce a delay in breasfeeding[14]. However, almost no hospitals in Korea have such guidelines tailored for the specific conditions of LPIs. Fourth, because the hospital stay of LPIs is shorter than that of premature infants under the gestational age of 34 weeks, the opportunity to provide breastfeeding education by health care providers is often inadequate[5]. Consequently, the prevalence of breastfeeding for LPIs was lower than that of prematures born at less than 34 weeks of gestational age $[5,11]$ and the 36 week LPIs who had the shortest stay in hospital had the lowest breastfeeding rate[10].

In light of addressing such issues, this study was conducted to identify breastfeeding practice of LPIs until 12 weeks after discharge, and predictors influencing breastfeeding at the 12th week after hospitalization. The rationale for establishing the 12th week after discharge as the time point of identifying the predictors for breastfeeding is based on a study done by Jang et al.[11]. Jang et al.[11] conducted a cross-sectional study to identify the predictors of breastfeeding practice in mothers from 1 to 6 months after discharge, reporting that the mothers' breastfeeding significantly decreased from three months after discharge, which suggested that better-organized longitudinal studies should be conducted to encompass a period from discharge to three months thereafter, in order to identify any changes in breastfeeding and to predict factors contributing to the exclusive breastfeeding.

\section{Methods}

\section{Study design and participants}

Participants of this study included 108 mothers whose newborns were hospitalized in neonatal intensive care units (NICUs) at two university hospitals in Korea. The mothers agreed to participate in this study after being provided a full explanation of the study purpose and data collection procedures. The two university hospitals recommended breastfeeding to the mothers. Both hospitals offered an educational program to mothers on how to extract and manage breast milk during their LPIs hospitalization in the NICU. The inclusion criterion was for the mothers to have a strong intention to breastfeed, assuming the health of their LPIs was satisfactory. Among the 108 mothers, two were excluded from this study because they withdrew themselves at the third and the eighth weeks after discharge, leaving results from the 106 participants for the final analysis. The exclusion criteria for mothers and LPIs were as follows: First, late preterm infants who showed some signs of growth retardation in the intrauterine environment, and who were at the lowest 10th percentile of birth weight; Second, infants who were born with serious respiratory problems and were using a ventilator; Finally, mothers who preferred formula feeding to breastfeeding.

\section{Data collection}

Data were collected from February to October 2013, by nurses who had more than 10 years of NICU experience in Korea. The data on LPIs, their mothers, and feeding-related characteristics were collected either by interviews with mothers on the day of discharge, or by reviewing of medical records one day prior to discharge. Nurses working in NICUs collected data on feeding method at home through telephone calls from the first week to the 12th week after discharge.

Based on the review of previous studies $[8,11,15,16]$ on predictors of breastfeeding practice for premature and LPIs, questionnaires were developed for this study, including characteristics of LPIs, their mothers, and feeding-related characteristics. Characteristics of LPIs included gestational age, the total length of hospitalization, gender, birth order, the number of births (singleton or twins), birth weight, and Apgar scores at one minute and five minutes after birth. Maternal characteristics included age, delivery types, employment, and mother's disease during pregnancy. Feeding-related characteristics included the time of hospitalization when the first feeding started and body weight at the first feeding, the total number of days for feeding during hospitalization, feeding intolerance of the late preterm and feeding methods during hospitalization.

Feeding methods at home were classified into the following three types: Exclusive breastfeeding, in which LPIs were fed exclusively with their mother's breast milk, mixed feeding, whereby a combination of breast milk and formula was used, and formula feeding, in which LPIs were exclusively formula fed.

\section{Ethical considerations}

Before data collection, this study received the Institutional Review 
Board approval (KNUH 2013-06-014). After the participants of this study were selected according to the inclusion and exclusion criteria, a full explanation of the study purpose was given to them, and written consent forms were collected. Because the study participants included premature infants, only the names of the premature infants and their mothers, as well as their medical record numbers were identified from medical records in order to confirm that mothers were legal representatives of the infants.

\section{Statistical analysis}

Data were analyzed using SAS version 9.1 (SAS Institute Inc., Cary, NC). Descriptive and inferential statistical analyses were performed in order to analyze demographic characteristics of LPIs, mothers, and feeding. As predictors for exclusive breastfeeding after discharge can be repeatedly measured over time, we used the Generalized Estimating Equations (GEE), a linear regression model that can be used to measure categorical dependent variables[17]. The dependent variable for the GEE model used an order scale (formula $=1$, mixed $=2$, breastfeeding $=3$ ). Also, the GEE model assumed that a link function was the multinomial distribution and the working correlation matrix was independent. The reason for selecting an independent working correlation matrix was that it was only available for multinomial models at SAS version 9.1. Fortunately, the GEE estimator is robust regardless of used correlation structure and the result is similar[17]. Correlations between the 1st week and the 2nd week, 1st week and 3rd week, 1st week and 4th week, 1st week and 8th week and 1st week and 12th week were $.74, .57, .49, .36$, and .34, respectively.

\section{Results}

\section{Demographic characteristics of the participants}

The gestational age of LPIs ranged from 20 LPIs at 34 weeks (18.9\%), 40 at 35 weeks (37.7\%), and 46 at 36 weeks (43.4\%). Infant boys accounted for $58.5 \%$ of LPIs, while the first child in birth order was $50.9 \%$, and singletons were $67.9 \%$. The average birth weight was 2,493 $\mathrm{g}$ with average Apgar scores at one minute and five minutes after birth being 6.96 and 8.31 respectively. The average length of hospitalization was 10.96 days. The average age of mothers was 33.19 years with $73.6 \%$ of mothers were unemployed, and $83.0 \%$ of mothers were ithout any diseases. Caesarean section accounted for $79.2 \%$ of all the delivery types (Table 1).

\section{Feeding characteristics during hospitalization}

The first feeding for LPIs started at 2.21 days after birth, on average, and the mean body weight of LPIs at the time of the first feeding was $2,426 \mathrm{~g}$. The average total number of days for feeding during hospitalization was 9.18 days. For feeding intolerance that was operationally defined

Table 1. Demographic Characteristics of Late Preterm Infants and their Mothers

$(\mathrm{N}=106)$

\begin{tabular}{|c|c|c|c|c|c|}
\hline Subject & Characteristics & Categories & $\mathrm{n}(\%)$ & $\mathrm{M} \pm \mathrm{SD}$ & Range (Min-Max) \\
\hline \multirow[t]{14}{*}{ Late preterm } & Gestational age & 34 weeks & 20 (18.9) & & \\
\hline & & 35 weeks & $40(37.7)$ & & \\
\hline & & 36 weeks & $46(43.4)$ & & \\
\hline & Gender & Male & $62(58.5)$ & & \\
\hline & & Female & $44(41.5)$ & & \\
\hline & Birth order & First-born & $50(50.9)$ & & \\
\hline & & Second-born & $40(37.7)$ & & \\
\hline & & Third-born & $12(11.3)$ & & \\
\hline & Infant plurality & Singleton & $72(67.9)$ & & \\
\hline & & Twin & $34(32.1)$ & & \\
\hline & Hospital stay (day) & & & $10.96 \pm 5.08$ & $3-28$ \\
\hline & Birth weight (g) & & & $2,493.40 \pm 365.48$ & $1,570-3,670$ \\
\hline & Apgar score (1 min) & & & $6.96 \pm 0.97$ & $3.0-9.0$ \\
\hline & Apgar score (5 min) & & & $8.31 \pm 0.88$ & $5.0-10.0$ \\
\hline \multirow[t]{7}{*}{ Mother } & Age (year) & & & $33.19 \pm 3.70$ & $26-43$ \\
\hline & Type of delivery & Vaginal & $22(20.8)$ & & \\
\hline & & Cesarean section & $84(79.2)$ & & \\
\hline & Employment & Yes & $28(26.4)$ & & \\
\hline & & No & 78 (73.6) & & \\
\hline & Maternal disease & Yes & $18(17.0)$ & & \\
\hline & & No & $88(83.0)$ & & \\
\hline
\end{tabular}


Table 2. Late Preterm Infants' Feeding Characteristics during Hospitalizatio

$(\mathrm{N}=106)$

\begin{tabular}{llccc}
\hline Characteristics & Categories & $\mathrm{n}(\%)$ & $\mathrm{M} \pm$ SD & Range (Min-Max) \\
\hline First feeding day & & & $2.21 \pm 1.68$ & $1-10$ \\
Body weight at first feeding (g) & & & $2,426.98 \pm 353.02$ & $1,620-3,480$ \\
Length of feeding period (day) & & $46(43.4)$ & $9.18 \pm 4.23$ & $3-25$ \\
Feeding intolerance & Yes & $60(56.6)$ & & \\
& No & $24(22.6)$ & & \\
Feeding method & Formula & $82(77.4)$ & & \\
& Mixed & $38(35.9)$ & \\
& Breastmilk $\geq$ Formula & $44(41.5)$ & \\
\hline
\end{tabular}

Table 3. Late Preterm Infants' Feeding Method and Comparison with Prevalence of Exclusive Breastfeeding

$(\mathrm{N}=106)$

\begin{tabular}{llccc}
\hline Time (week) & Categories & $\mathrm{n}(\%)$ & Difference (\%)* & $p$ \\
\hline 1 & Exclusive BF & $6(5.7)$ & & \\
& Mixed feeding & $71(67.0)$ & & \\
& Formula feeding & $29(27.3)$ & & .580 \\
2 & Exclusive BF & $8(7.6)$ & 1.9 & \\
& Mixed feeding & $56(52.8)$ & & .298 \\
& Formula feeding & $42(39.6)$ & & \\
3 & Exclusive BF & $10(9.4)$ & 3.7 & .206 \\
& Mixed feeding & $41(38.7)$ & & \\
4 & Formula feeding & $55(51.9)$ & & .009 \\
& Exclusive BF & $11(10.4)$ & 4.7 & \\
& Mixed feeding & $34(32.1)$ & & .002 \\
& Formula feeding & $61(57.5)$ & & \\
& Exclusive BF & $18(17.0)$ & 11.3 & \\
& Mixed feeding & $17(16.0)$ & & \\
& Formula feeding & $71(67.0)$ & & \\
& Exclusive BF & $21(19.8)$ & 14.1 & \\
& Mixed feeding & $13(12.3)$ & & \\
& Formula feeding & $72(67.9)$ & & \\
\end{tabular}

*Difference between exclusive breast-feeding of each week and that of 1st week. $\mathrm{BF}=$ Breast-Feeding.

in this study as the late preterm having difficulties in feeding more than 4 times a day, mothers of $56.6 \%$ of the LPIs reported having no difficulties. Feeding method for LPIs during hospitalization was either formula and mixed feeding. Mixed feeding was estimated at $77.4 \%$ of all methods of feeding, which showed that when LPIs were given mixed feeding, they were more inclined to use formula than breast milk (Table 2).

\section{Late preterm infants' feeding method until 12 weeks}

The rate of exclusive breastfeeding for LPIs continuously increased as $5.7 \%, 7.6 \%, 9.4 \%, 10.4 \%, 17.0 \%$, and $19.8 \%$ for week of $1,2,3,4,8$, and 12 respectively, so did formula feeding from $27.3 \%$ at the first week to $67.9 \%$ at the 12th week. Contrarily, the mixed method of feeding decreased from $67.0 \%$ at the first week to $12.3 \%$ at the 12 th week (Table 3, Figure 1).

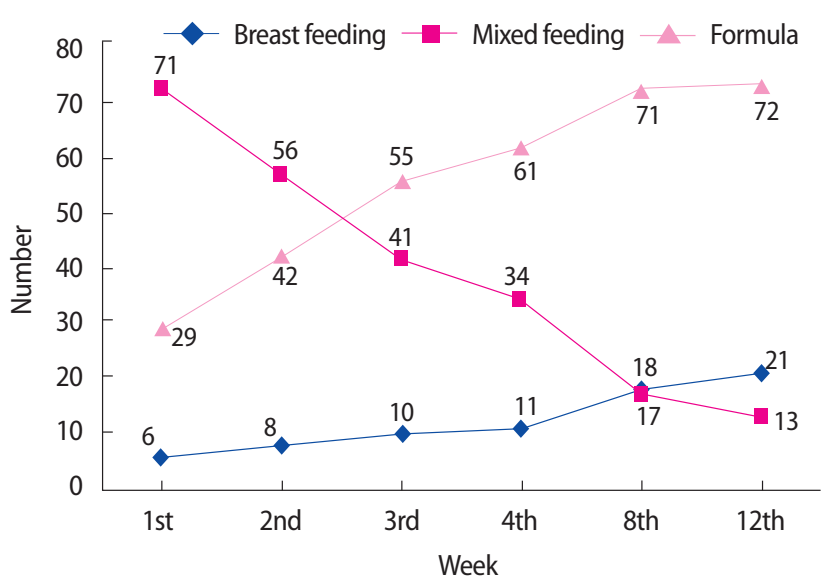

Figure 1. Comparison of late preterm infants' feeding methods according to time elapse after discharge.

Of 71 mothers $(67.0 \%)$ who used mixed feeding for the first week after discharge, two mothers turned to breastfeeding and 13 mothers to formula feeding. Therefore, mothers who had used the mixed feeding turned to exclusive breastfeeding or formula feeding over time. Unexpectedly, the ratio of formula feeding was higher than that of exclusive breastfeeding over time.

The rate of exclusive breastfeeding showed no significant difference between the 1st week and the 2nd week ( $p=.580)$, 1st week and 3rd week $(p=.298)$ and 1 st week and 4 th week $(p=.206)$. However, the difference of exclusive breastfeeding was statistically significant between the 1st week and the 8th week $(p=.009)$ and the 1st week and the 12th week $(p=.002)$.

\section{Predictors related to exclusive breast-feeding performance in late preterm}

Predictors for exclusive breastfeeding at the time of the 12th week after discharge were interpreted as two orders, in which formula feeding was changed into mixed feeding and mixed feeding was changed into 
Table 4. Predictors Related to Exclusive Breast-Feeding for Late Preterm Infants

$(\mathrm{N}=106)$

\begin{tabular}{|c|c|c|c|c|c|c|}
\hline Variables & Estimate* & SE & $z$ & $p$ & $\mathrm{OR}^{\dagger}$ & $95 \% \mathrm{Cl}$ \\
\hline \multicolumn{7}{|l|}{ Type of delivery } \\
\hline Cesarean section & 1.00 & & & & & \\
\hline \multicolumn{7}{|l|}{ Feeding intolerance } \\
\hline Yes & 1.00 & & & & & \\
\hline No & 1.11 & 0.45 & 2.48 & .013 & 3.03 & $1.26-7.25$ \\
\hline Formula & 1.00 & & & & & \\
\hline Mixed & 2.06 & 0.47 & 4.43 & $<.001$ & 7.84 & $3.15-19.53$ \\
\hline
\end{tabular}

*Estimate of each independent variables was adjusted for LPIs' characteristics (gestational age, gender, birth rank, infant plurality, hospital stay, birth weight, 1 min-Apgar score and 5-min Apgar score), mothers' characteristics (age, employment and maternal disease) and feeding characteristics (first feeding day, body weight at first feeding, and length of feeding period); ${ }^{\top}$ The dependent variable for GEE model was used order scale (formula $=1$, mixed $=2$, breastfeeding $=3$ ).

exclusive breastfeeding. The results showed that mothers who delivered through the vaginal procedure changed their feeding method 2.96 times more into mixed feeding and 2.96 times more into exclusive breastfeeding than those who delivered through Cesarean-section $(\mathrm{OR}=2.96,95 \%$ $\mathrm{CI}=1.07-8.14)$. In addition, LPIs who did not have any feeding intolerance were 3.03 times more often to be fed through mixed feeding and 3.03 times more often, breastfed exclusively than those who had feeding intolerance ( $\mathrm{OR}=3.03,95 \% \mathrm{CI}=1.26-7.25)$. Finally, LPIs who were fed mixed feeding during hospitalization were 7.84 times more likely to change to mixed feeding and exclusive breastfeeding than those who were fed formula $(\mathrm{OR}=7.84,95 \% \mathrm{CI}=3.15-19.53)$ (Table 4).

\section{Discussion}

Breastfeeding newborns until the age of two years plays an important role in providing nutritional requirements necessary for the proper growth and development of infants[12]. Breast milk has been scientifically proved to contain immunological and anti-infective factors that prevent intestinal diseases[18]. However, neither the exact number of breastfeeding of late preterms, nor standardized guidelines for age-appropriate breastfeeding have been established yet at the governmental level in Korea. Therefore, to provide baseline information to develop breastfeeding education tailored for the particular needs of LPIs, this study was done to identify breastfeeding practice with LPIs for 12 weeks after discharge, as well as predictors influencing exclusive breastfeeding practice at the 12th week after discharge.

One of the most important results of this study showed that $77.4 \%$ of LPIs were fed with the mixed feeding method during hospitalization. This was consistent with other studies conducted with full-term in- fants[19]. Jang and Kim[19] reported that nearly 20\% of full-term newborns were fed exclusively with breast milk, while $80 \%$ were fed with the mixed feeding method. The general guidelines of educational programs for breastfeeding provided by the institutions are that nurses recommend supplementing breastfeeding with formula both for full-term and LPIs when mothers cannot fully feed colostrum to newborns due to the amount of colostrum not being sufficient. This led the majority of infants to be discharged with an experience of mixed feeding. In addition, because of LPIs' lack of sucking and swallowing power, it is not only difficult to start breastfeeding within one hour after birth, but also to maintain exclusive breastfeeding until the time of discharge. This results in LPIs being discharged with mixed feeding, rather than exclusive breastfeeding, much more frequently than full-term infants[20,21].

Exclusive breastfeeding and formula feeding steadily increased, and mixed feeding decreased after discharge. This result is derived from the fact that the mothers who continued mixed feeding, changed to exclusive breastfeeding or formula feeding, moreover the change rate of mixed feeding to formula was higher than that to exclusive breastfeeding from mixed feeding. About $10.4 \%$ of mothers who breastfed their LPIs at the 4 th week after discharge was far lower than what is recommended by World Health Organization[12]. This lower rate of breastfeeding practice for LPIs compared to full-term infants can be explained by the physiological effects of late preterm birth itself, which is considered the most important contributing factor for the failure of breastfeeding[22]. A multicenter study done by Medoff-Cooper et al.[23] with 802 LPIs at 14 hospitals in the United States and Canada, reported that although LPIs whose gestational age was close to full-term, they still had problems with feeding intolerance due to physiological immaturity, causing impairment of masseteric and sucking functions. A second reason for the lower 
rate of breastfeeding in this study can be explained by showing that mothers who gave birth to LPIs usually had premature labor due to health conditions caused by pregnancy induced hypertension, diabetes mellitus, or obesity. These health conditions inhibit secretion of prolactin in mothers, delaying breastfeeding compared to mothers who deliver full-term newborns $[13,16]$. The third reason is that most guidelines for breastfeeding used in clinical settings in Korea have been developed, focusing on mothers with full-term newborns, leaving mothers with LPIs with inadequate instruction. Furthermore, the extent to which educational programs for breastfeeding are given to mothers with LPIs often differ from unit to unit within individual hospitals. The final reason is that as the American Academy of Pediatrics[23] have reported that LPIs over the gestational age of 35 weeks were 2.2 times more likely to show jaundice when they were breastfed than full-term infants, resulting in rehospitalization due to jaundice or other infectious disease[9]. Consequently, some health care providers may be hesitant to recommend breastfeeding for LPIs as the best method. In light these issues, nurses should carefully assess LPIs' clinical conditions, such as hypoglycemia, hypothermia, respiratory difficulty, or feeding intolerance, all of which may affect the desirability of breast feeding.

Finally, the factors that predict exclusive breastfeeding practice at the 12th weeks after discharge showed that mothers who had a vaginal delivery changed from formula feeding to mixed feeding or mixed feeding to exclusive breastfeeding 2.96 times more often than mothers who had Cesarean-section. Plus, late preterms who did not have any feeding intolerance were breastfed 3.03 times more than infants who had feeding intolerance, and LPIs who were fed mixed feeding during hospitalization had 7.84 times more exclusive breastfeeding than those who were fed formula. This result was consistent with another study[11]. Jang et al.[11] reported factors influencing breastfeeding for LPIs were the type of delivery and feeding method during hospitalization. Special consideration should be given to the effectiveness of breastfeeding during hospitalization, which can affect the continuation of breastfeeding after hospitalization up until the 12th week. Along with the provision of proper nursing care for health problems for both mothers and infants, education is one of the most important tools that nurses can use to promote breastfeeding LPIs during and after hospitalization. Unlike guidelines for full-term newborns who can be fed right after birth, the guidelines for LPIs who have to be hospitalized for a certain period of time should include special measures for stimulating the lactation process and the production of breast milk.
Breastfeeding LPIs in this study increased continuously from the 1st to the 12th week after discharge, but many mothers changed from mixed breastfeeding to formula feeding rather than breastfeeding exclusively. In addition, breastfeeding at the time of the 12th week after discharge was a major predictor indicating mothers who had breastfed their infants during hospitalization would be more likely to continue after hospitalization than their counterparts. These findings suggest that further research is needed to address the issues of developing more effective educational guidelines for breastfeeding, tailored to the specific needs of LPIs, with a particular focus on how to convert mothers who feed their infants with mixed feeding after hospitalization, to change to exclusive breastfeeding.

Several limitations of this study may be worthy of discussion. First, this study included only two hospitals in Korea and the results cannot be considered definitive. However, considering the importance of breastfeeding for the LPIs, these hospitals were carefully selected to represent strong support for promoting breastfeeding in Korea. To compare the variations of educational guidelines for breastfeeding, further study is needed to include general practices of breastfeeding at all Korean hospitals, not just at the hospitals in this study. Another limitation of this study is that it did not include underlying psychological factors that could play a key role in predicting successful practice of breastfeeding in mothers with LPIs, such as self-efficacy or empowerment. Finally, this study contained a relatively small sample size of LPIs and their mothers. In order to confirm of the findings in this work, more replicated studies are needed to include different mother-infant dyads at different clinical settings, as well as studies to collect data on breastfeeding appropriate for government use.

\section{Conclusion}

Breast milk contains nutritional substances essential for LPIs. For this reason, breastfeeding is recommended as the healthiest source of nutrition for LPIs. Consequently, our study was conducted to identify breastfeeding LPIs during the 1st to the 12th week after discharge, as well as factors influencing exclusive breastfeeding practice at the 12th week after discharge. Exclusive breastfeeding in LPIs had continuously increased for the period of 12 weeks after they were discharged from the hospital, and the mixed feeding method at the time of discharge changed to formula feeding exclusively over time, which indicated that educational programs to promote breastfeeding should be focused on how to con- 
tinue exclusive breastfeeding for at least 12 weeks after discharge. Furthermore, mothers' breastfeeding during hospitalization affected the practice of breastfeeding at 12th week after discharge. Therefore, special consideration in education should be given to mothers with LPIs in addition to more effective methods of breastfeeding at the hospital and at home. The significance of this study includes an attempt to provide practical information on appropriate times and directions for breastfeeding interventions targeted for mothers who have not been instructed properly, due to a lack of established guidelines in Korea.

\section{Conflict of Interest}

No potential conflict of interest relevant to this article was reported.

\section{Acknowledgements}

This work was supported by the Korea Research Foundation (KRF) grant funded by the Korea government (MEST) (2009-0070334).

\section{References}

1. World Health Organization. International statistical classification of diseases and related health problems Volume 2 Instruction Manual [Internet]. Geneva: World Health Organization; 2010. [cited 2014 May 10]. Available from: http://www.who.int/classification/icd/en/.

2. Engle WA. A recommendation for the definition of "late preterm" (near term) and the birth weight-gestational age classification system. Seminars in Perinatology. 2006;30(1):2-7. http://dx.doi.org/10.1053/ j.semperi.2006.01.007

3. Statistics Korea. 2012 live births by period of pregnancy [Internet]. Seoul: Statistics Korea; 2015 [cited 2014 March 10]. Available from: http://kosis.kr/eng/statisticsList/statisticsList_01List.jsp?vwcd=MT_ ETITLE\&parentId=A\#SubCont.

4. Demirci JR, Sereika SM, Bogen D. Prevalence and predictors of early breastfeeding among late preterm mother-infant dyads. Breastfeeding Medicine. 2013;8(3):277-285. http://dx.doi.org/10.1089/bfm.2012.0075

5. Colaizy TT, Morris FH. Positive effect of NICU admission on breastfeeding of preterm US infants in 2000 to 2003. Journal of Perinatology. 2008;28(7):505-510. http://dx.doi.org/10.1038/jp.2008.32

6. Kim MJ. Readmission of late preterm infants after discharge from nursery. Korean Journal of Pediatrics. 2009;52(8):888-892. http:// dx.doi.org/10.3345/kjp.2009.52.8.888

7. Na JY, Park N, Kim ES, Lee HJ, Shim GH, Lee JA, et al. Short-term clinical outcomes of late preterm infants. Korean Journal of Pediatrics. 2009;52(3):303-309. http://dx.doi.org/10.3345/kjp.2009.52.3.303

8. Cleaveland K. Feeding challenges in the late preterm infant. Neonatal Network. 2010;29(1):37-41. http://dx.doi.org/10.1891/0730-0832.29.1.37

9. Tomashek KM, Shapiro-Mendoza CK, Weiss J, Kotelchuck M, Barfield W, Evans S, et al. Early discharge among late preterm and term newborns and risk of neonatal morbidity. Seminars in Perinatology. 2006;30(2):61-68. http://dx.doi.org/10.1053/j.semperi.2006.02.003

10. Jang GJ. Comparing factors associated with breastfeeding in late preterm infants of different gestational ages. Child Health Nursing Research. 2015;21(4):302-310. http://dx.doi.org/10.4094/chnr.2015.21.4.302

11. Jang GJ, Lee SL, Kim HM. Breast feeding rates and factors influencing breast feeding practice in late preterm infants: comparison with preterm born at less than 34 weeks of gestational age. Journal of Korean Academy of Nursing. 2012;42(2):181-189. http://dx.doi.org/10.4040/ jkan.2012.42.2.181

12. World Health Organization. Indicators for assessing infant and young child feeding practices part 1 Definitions [Internet]. Geneva: World Health Organization; 2008 [cited 2014 May 10]. Available from: http:// www.who.int/maternal_child_adolescent/documents/9789241596664/ en/.

13. Hurst NM, Meier PP. Breastfeeding the preterm infant. In: Riordan J, Wambach K, editors. Breastfeeding and human lactation. 4th ed. MA; Jones and Bartlett: 2010. p. 425-470.

14. Ludwig SM. Oral feeding and the late preterm infant. Newborn and Infant Nursing Reviews. 2007;7(2):72-75. http://dx.doi.org/10.1053/ j.nainr.2007.05.005

15. Howe TH, Sheu CF, Hinojosa J, Lin J, Holzman IR. Multiple factors related to bottle-feeding performance in preterm infants. Nursing Research. 2007;56(5):307-311. http://dx.doi.org/10.1097/01.NNR.00002 89498.99542.dd

16. Walker M. Breastfeeding the late preterm infant. Journal of Obstetric, Gynecologic, and Neonatal Nursing. 2008;37(6):692-701. http://dx.doi. org/10.1111/j.1552-6909.2008.00293.x

17. Liu S, Dixon J, Qiu G, Tian Y, McCorkle R. Using generalized estimating equations to analyze longitudinal data in nursing research. Western Journal of Nursing Research. 2009;31(7):948-964. http://dx.doi.org/ $10.1177 / 0193945909336931$

18. Callen J, Pinelli J. A review of the literature examining the benefits and 
challenges, incidence and duration, and barriers to breastfeeding in preterm infants. Advances in Neonatal Care. 2005;5(2):89-92. http:// dx.doi.org/10.1016/j.adnc.2004.1012.1003

19. Jang GJ, Kim SH. Effects of breast-feeding education and support services on breast-feeding rates and infant's growth. Journal Korean Academy of Nursing. 2010;40(2):277-286. http://dx.doi.org/10.4040/ jkan.2010.40.2.277

20. Ayton J, Hansen E, Quinn S, Nelson M. Factors associated with initiation and exclusive breastfeeding at hospital discharge: late preterm compared to 37 week gestation mother and infant cohort. International Breastfeeding Journal. 2012;7(1):1-6. http://dx.doi.org/10.1186/17464358-7-16
21. Medoff-Cooper B, Holditch-Davis D, Verklan MT, Fraser-Askin D, Lamp J, Santa-Donato A, et al. Newborn clinical outcomes of the AWHONN late preterm infant research-based practice project. Journal of Obstetric, Gynecologic, and Neonatal Nursing. 2012;41(6):774785. http://dx.doi.org/10.1111/j.1552-6909.2012.01401.x

22. Nyqvist KH. Breastfeeding preterm infants. In: Genna CW, editor Supporting sucking skills in breastfeeding infants. MA; Jones and Bartlett: 2008. p. 153-180.

23. American Academy of Pediatrics. Management of hyperbilirubinemia in the newborn infant 35 or more weeks of gestation. Pediatrics. 2004; 114(1):297-316. http://dx.doi.org/10.1542/peds.114.1.297 